\title{
Synthesis, Properties and Chemical Modification of a Persistent Triisopropylsilylethynyl Substituted Tri(9-anthryl)methyl Radical
}

\author{
Tomohiko Nishiuchi, ${ }^{a *}$ Daisuke Ishii, ${ }^{a}$ Seito Aibara, ${ }^{a}$ and Takashi Kubo ${ }^{a, b *}$
}

In studies aimed at developing new organic spin materials, we prepared a triisopropylsilylethynyl substituted tri(9-anthryl)methyl (TAntM) radical. The TIPS-ethynyl group in this radical effectively suppress its reactivity, resulting in extremely high stability in air for at least one month. Chemical modification of the radical using [4+2] Diels-Alder reaction proceeds even at room temperature. Because harsh conditions and metal-catalyzed reactions are not required this post-modification strategy should be highly versatile for use in constructing unique spin-labelled molecules.

Highly persistent neutral organic radicals have attracted great attention recently. Various radicals of this type have been synthesized to explore their physical and photophysical properties as well as utilization as functional materials in organic magnets, ${ }^{[1]}$ batteries, ${ }^{[2]}$ magnetic resonance imaging systems, ${ }^{[3]}$ doublet emission based light emitting diodes ${ }^{[4]}$ and dynamic nuclear polarization (DNP) studies. ${ }^{[5]}$ To enable them to be highly persistent, organic radicals usually contain nitrogen or oxygen atoms to provide thermodynamic stability. ${ }^{[6,7]}$ Alternatively, the stabilities of purely hydrocarbon radicals can be enhanced by incorporating bulky substituents at positions bearing high spin densities to inhibit their reactivities ${ }^{[8,9]}$ (Figure 1). For example, perchlorotriphenylmethyl (PTM) ${ }^{[10]}$ and tetrathiatriarylmethytl $\left(\mathrm{TAM}^{[11]}\right.$ radicals are well known persistent aromatic hydrocarbon radicals that gain stability from kinetic protection of their spin-localized central $\mathrm{sp}^{2}$ carbons by halogen or sulfur atoms. Owing to the presence of embedded heteroatoms at high spin density locations, PTM and TAM radicals have relatively large $g$-anisotropies. However,

\footnotetext{
a. Department of Chemistry, Graduate School of Science, Osaka University, Toyonaka, Osaka 560-0043, Japan.E-mail: nishiuchit13@chem.sci.osaka-u.ac.jp b. Innovative Catalysis Science Division, Institute for Open and Transdisciplinary Research Initiatives, (ICS-OTRI), Osaka University, Suita, Osaka 565-0871, Japan.
}

persistent non-heteroatom embedded hydrocarbon radicals with small $g$-anisotropies are the most ideal candidates for use in various spintronic materials. ${ }^{[12]}$ Unfortunately, the library of stable hydrocarbon radicals not containing heteroatoms remains small because of structural limitations as well as synthetic issues.

We recently prepared the purely hydrocarbon mesitylsubstituted tri(9-anthryl)methyl (TAntM) radical, ${ }^{[8]}$ in which the central $\mathrm{sp}^{2}$ carbon is sterically protected by three 9-anthryl groups. In addition, we demonstrated that TAntM radical displays extremely high persistence, undergoing only slight decay after more than a month in air-saturated solution (Figure 2). Because no heteroatoms are present, the TAntM radical is expected to have small $g$-anisotropy and, therefore, its functionalized derivatives should be attractive targets in efforts aimed at developing and applying new organic spin materials.

In the investigation described below, whose overall goal is to increase the scope and versatility of hydrocarbon radicals for organic-based materials, we prepared the TAntM radical $\mathbf{1}$ containing a triisopropylsilyl (TIPS) ethynyl group and assessed its properties and chemical reactions. The results show that TIPS-ethynyl group in $\mathbf{1}$ effectively enhances its stability in air. Moreover, we demonstrated that modification of 1 by a DielsAlder reaction can be utilized to produce the related tetraphenylphenyl substituted TAntM radical 2.

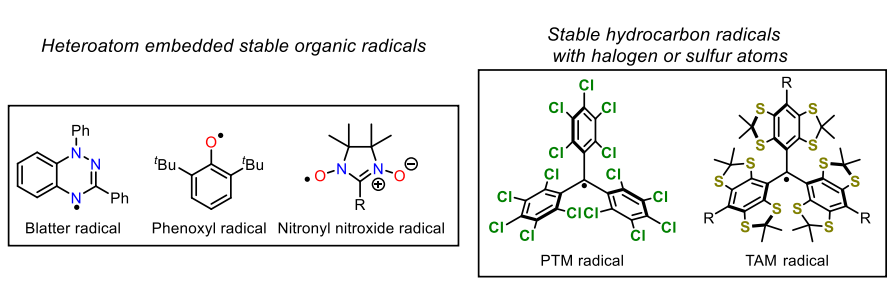

Figure 1. Stable neutral organic radicals embedded with heteroatoms for gaining thermodynamic stability (upper) and stable hydrocarbon radicals with halogen or sulphur atoms for gaining kinetic stability (below). 


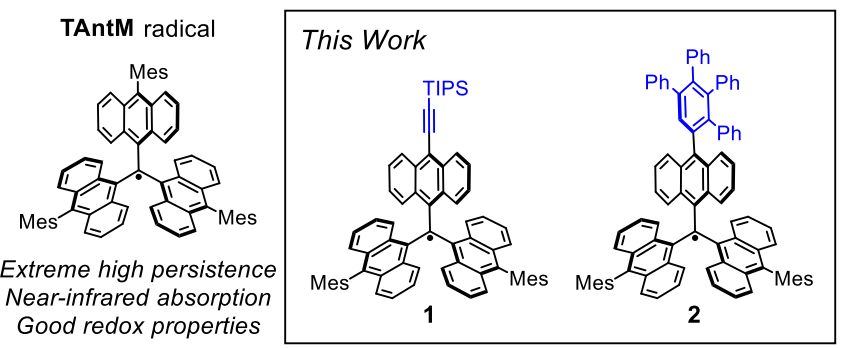

Figure 2. The structure of TAntM radical (left) and functionalized TAntM radicals $\mathbf{1}$ and $\mathbf{2}$ (right).

The route employed to synthesize $\mathbf{1}$, shown in Scheme 1, begins with the 10-alkylidene-9-anthrone derivative 3 which was prepared using a reported procedure. ${ }^{[8 \mathrm{e}]}$ Reaction of $\mathbf{3}$ with TIPS-ethynyl lithium generated the corresponding alkynylcarbinol 4 in high yield (92\%). Treatment of 4 with tin (II) chloride dihydrate then efficiently $(90 \%)$ produced the target radical 1 . Although the steric hindrance provided by the TIPSethynyl unit should be less than that by the mesityl group, it is worth noting that the radical $\mathbf{1}$ has a stability that matches that of TAntM radical, ${ }^{[13]}$ even to the extent of allowing purification by silica gel chromatography in air.

The structure of radical 1 was unambiguously confirmed by using X-ray crystallographic analysis (Figure 3, Figure S1). Analysis of the X-ray data shows that radical 1 has a $\mathrm{C}-\mathrm{C}$ bond distance between the central $\mathrm{sp}^{2}$ carbon $\mathrm{C} 1$ and the anthryl carbon C2 (C1-C2) of 1.452(3) $\AA$, which is slightly shorter than

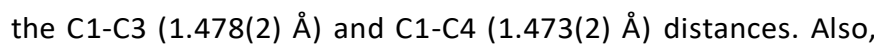
the anthryl unit twist angle $\theta 1\left(37.4^{\circ}\right)$ is smaller than $\theta 2\left(48.5^{\circ}\right)$ and $\theta 3\left(47.5^{\circ}\right)$, indicating that the distribution of unpaired electron on the TIPS ethynyl-substituted anthryl unit is slightly larger than on the other mesityl-substituted anthryl units.

Radical 1 has a $C_{2}$-like structure in the crystal state. Quantum chemical calculations on its TMS-analog $\mathbf{1}^{\prime}$ show that it has only a slight spin distribution on ethynyl unit (Figure 4a, Figure S3). Yet, the ESR spectrum of 1 contains a narrow peak (Figure 4b), which has a width that is identical to the peak in the spectrum of TAntM radical, which has $D_{3}$ symmetry. Thus, the spin distribution of $\mathbf{1}$ is similar to that of TAntM radical because the anthryl units can undergo ready rotation in solution.

The UV-vis-NIR spectrum of $\mathbf{1}$ is similar to that of TAntM radical (Figure 5a). The differences consist of a slight red-shift of maxima from those of TAntM radical in the visible region (600 $>620 \mathrm{~nm}$ ) and near-infrared region (1020 -> $1100 \mathrm{~nm}$ ) caused by the presence of the TIPS-ethynyl unit. These effects are also reflected in the results of TD-DFT calculations (Figure S4).

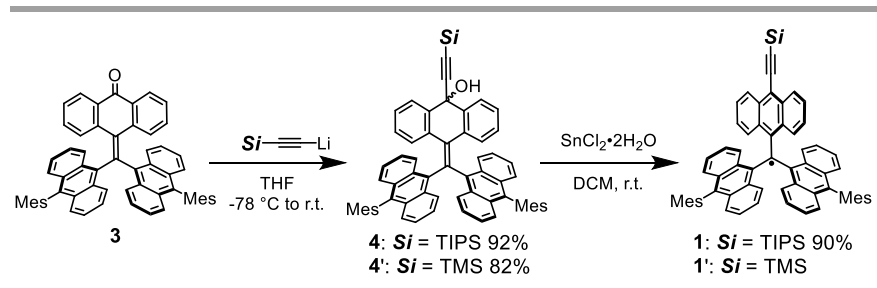

Scheme 1 . Routes for synthesis of $\mathbf{1}$ and $\mathbf{1}^{\prime}$.

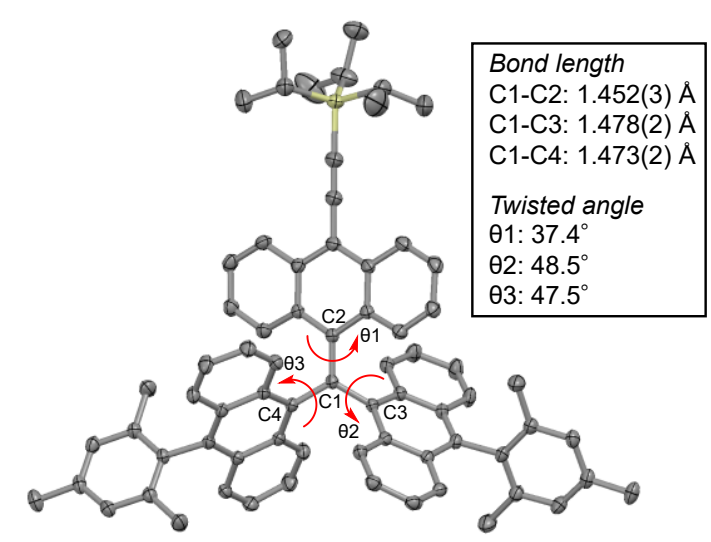

Figure 3. Plot of single crystal X-ray data of $\mathbf{1}$ and its structural factors (bond lengths and twist angles). Protons are omitted for clarity.

The effect of TIPS-ethynyl substitution is also seen in the redox properties of radical $\mathbf{1}$. The cyclic voltammogram (CV) of $\mathbf{1}$ contains reversible oxidation and reduction waves at $E^{\mathrm{Ox}_{1 / 2}}=-$ $0.15 \mathrm{~V}$ and $E^{\mathrm{Re}_{1 / 2}}=-1.35 \mathrm{~V}$, respectively (Figure $5 \mathrm{~b}$ ). In contrast, $\mathrm{CV}$ waves of TAntM radical $\left(E^{\mathrm{Ox}}{ }_{1 / 2}=-0.19 \mathrm{~V}\right.$ and $E^{\mathrm{Re}_{1 / 2}}=-1.48 \mathrm{~V}$, Figure S5) show that oxidation and reduction potentials are slightly positively shifted by the TIPS-ethynyl unit.

The stability of radical $\mathbf{1}$ in an air-saturated solution was assessed by monitoring the decay of the absorption maximum at $620 \mathrm{~nm}$. Surprisingly, no significant decomposition was observed to take place for at least one month (Figure S6).
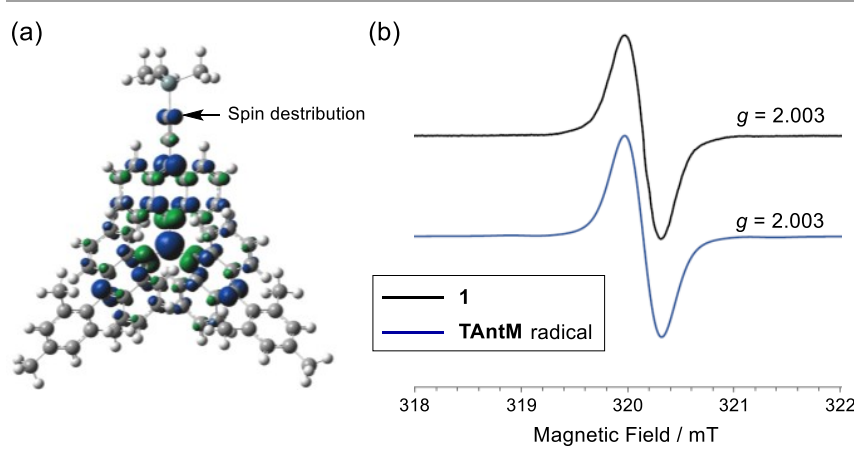

Figure 4. (a) Spin density map of $\mathbf{1}^{\prime}$ (UBLYP/6-31G**//WB97XD/6$31 \mathrm{G}^{* *}$ ). (b) ESR spectra of $\mathbf{1}$ (black line) and TAntM radical (blue line) in toluene.

(a)

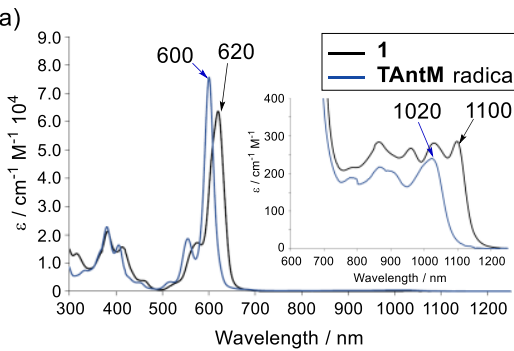

(b)

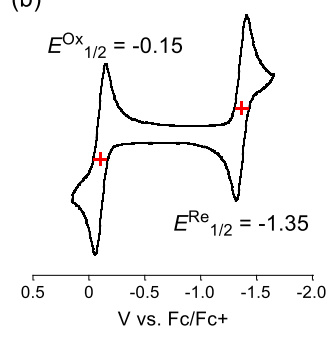

Figure 5. (a) UV-vis-NIR spectra of 1 and TAntM radical in $\mathrm{CH}_{2} \mathrm{Cl}_{2}$ (inset: expansion from 600 to $1250 \mathrm{~nm}$ ). (b) Cyclic voltammograms of 1 and TAnt $M$ radical. Measurement conditions: $0.1 \mathrm{M}^{n} \mathrm{Bu}_{4} \mathrm{NPF}_{6}$ in $\mathrm{CH}_{2} \mathrm{Cl}_{2}$, scan rate $=100 \mathrm{mV} \mathrm{s}^{-1}$. 
(a)

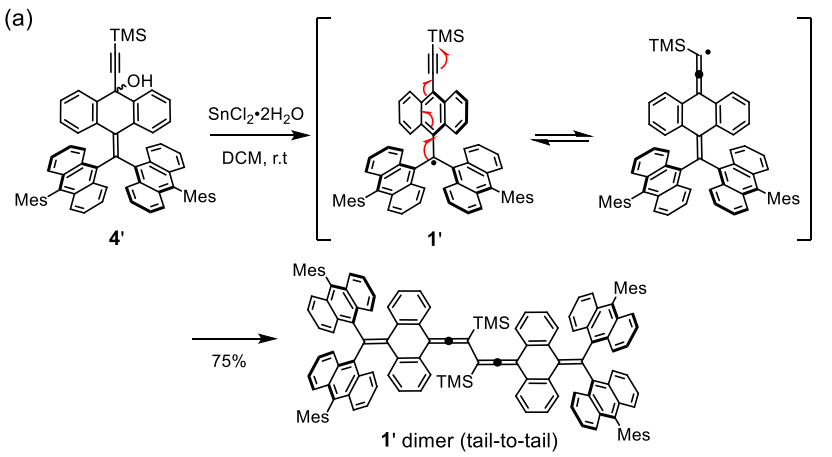

(b)

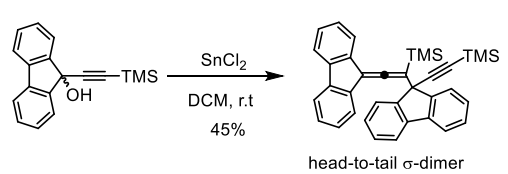

Scheme 2. (a) Reaction of $\mathbf{1}^{\prime}$ to form $\mathbf{1}^{\prime}$ dimer. (b) Reaction of the TMSethynyl substituted fluorenyl radical. ${ }^{[15]}$

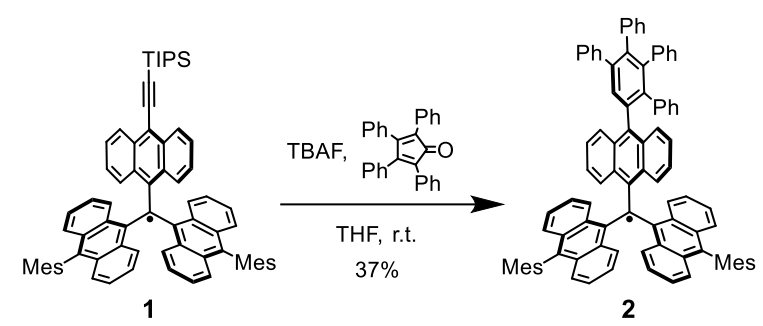

Scheme 3. Diels-Alder reaction of $\mathbf{1}$ to form radical 2.

Although the steric protection provided by its TIPS-ethynyl unit is less than that of a mesityl group, $\mathbf{1}$ has a stability that is comparable with that of TAntM radical. To ascertain reason(s) for this persistence, the related radical $\mathbf{1}^{\prime}$ containing a smaller TMS-ethynyl substituent was prepared (Scheme 1). The solution of formed 1' has a green color initially, but the color slowly changes to red along with formation of an orange precipitate. Unfortunately, a single crystal of $\mathbf{1}^{\prime}$ suitable for X-ray analysis can not be obtained. However, ${ }^{1} \mathrm{H}$ - and ${ }^{13} \mathrm{C} N M R, M S$, and IR analysis showed that in solution $\mathbf{1}^{\prime}$ efficiently reacts to generate an allene-type tail-to-tail 1' dimer in 75\% (Scheme 2a and Figure S7-9). Quantum chemical calculations indicate that the $\sigma$-dimer is energetically highly favorable $\left(-25.5 \mathrm{kcal} \mathrm{mol}^{-1}\right.$, Figure S10). For comparison purposes, the TMS-ethynyl substituted fluorenyl radical reacts to only form the asymmetric head-totail $\sigma$-dimer (allene-ethynyl) (Scheme $2 b)^{[14]}$ probably because the fluorenyl moiety is sterically less hindered than the TAntM unit. The combined results indicate that the TIPS-ethynyl unit in 1 stabilizes the radical both through a steric effect and electronically by delocalization of the unpaired electron.

Finally, the chemical reactivity of radical 1 was probed to evaluate its versatility in the preparation of new organic spin materials. Direct introduction of radical group in a molecular skeleton is an attractive strategy for creation of spin-based materials. The use of nitronyl nitroxide radical[15] exemplifies this approach.

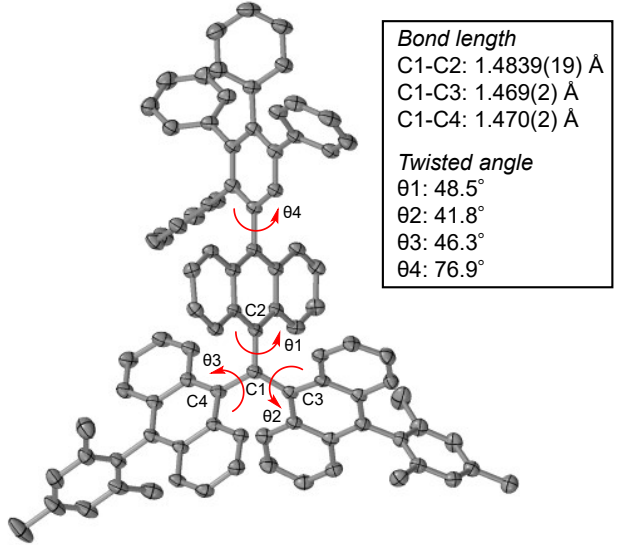

Figure 6. Plot of single crystal X-ray data of $\mathbf{2}$ and its structural factors (bond lengths and twist angles). Protons are omitted for clarity.

However, to use trityl-based persistent hydrocarbon radicals in this strategy, a functional group would need to be introduced before generating the radical (pre-modification) so that rapid decomposition can be avoided. ${ }^{[16]}$ To explore this strategy, we examined reactions of radical 1 involving in situ removal of the silyl group using TBAF and subsequent [4+2] Diels-Alder reaction using tetraphenylcyclopentadienone (tetracyclone) (Scheme 3). We observed that this process leads to formation of the tetraphenylphenyl substituted TAntM radical $\mathbf{2}$ in $37 \%$ yield. Surprisingly, although Diels-Alder reactions usually require relatively high temperatures $\left(>100^{\circ} \mathrm{C}\right)$, the one involved in generation of $\mathbf{2}$ takes place at room temperature.

Significantly, tetraphenylphenyl substituted radical $\mathbf{2}$ has almost the same properties as the TAntM radical. X-ray crystallographic analysis of $\mathbf{2}$ shows that its C1-C2 bond length is longer and the $\theta 1$ twist angle is larger than that of $\mathbf{1}$ (Figure 6, Figure S2), making it have close to the $D_{3}$ propeller structure of TAntM radical. In fact, UV-vis and ESR spectra, and CV of $\mathbf{2}$ are almost identical to those of TAntM radical (Figure S11-13). Due to steric bulkiness provided by the tetraphenyphenyl (TPB) unit, the twist angle $\theta 4$ between anthryl and TPB units is $76.9^{\circ}$. As a result, the stability of $\mathbf{2}$ is also comparable to that of TAntM radical and 1 (Figure S14). This result indicates the radical 2 retains the properties of the TAntM radical.

In summary, in the study described above, we synthesized TIPS-ethynyl substituted TAntM radical 1 and evaluated its properties. The results of studies with the analogous TMSethynyl substituted TAntM radical $\mathbf{1}^{\prime}$ gave important information that has led to an understanding of the stability/reactivity of the ethynyl moiety. Post-modification of radical 1 employing [4+2] Diels-Alder reaction with tetracyclone was found to occur effectively even at room temperature. In contrast to approaches often utilized to introduce radical groups into other molecules, the strategy developed in this effort avoids harsh reaction conditions and metal-catalyzed reactions that lead to decomposition and contamination with residual metals. In addition, tetracyclone, a model reactant in this study, can be readily modified such as by attaching emissive 
or amphiphilic components. Therefore, the facile postmodification process involving a room-temperature Diels-Alder reaction could become a highly versatile method to introduce the TAntM radicals into substances used as organic spinlabelled materials. Further development of development of this overall strategy is ongoing.

This study was supported by Grant-in-Aid for Scientific Research (C) (JSPS KAKENHI Grant Number JP20K05475). Quantum chemical calculations were performed at the Research Center for Computational Science, Okazaki, Japan.

\section{Conflicts of interest}

There are no conflicts to declare.

\section{Notes and references}

1 (a) M. Kinoshita, P. Truck, M. Tamura, K. Nozawa, D. Shiomi, Y. Nakazawa, M. Ishikawa, M. Takahashi, K. Awaga, T. Inabe, Y. Murayama, Chem. Lett. 1991, 20, 1225.; (b) Y. Nakazawa, M. Tamura, N. Shirakawa, D. Shiomi, M. Takahashi, M. Kinoshita, M. Ishikawa, Phys. Rev. B. 1992, 46, 8906.; (c) T. Sugawara, M. M. Matsushita, A. Izuoka, N. Wada, N. Takeda, M. Ishikawa, J. Chem. Soc. Chem. Commun. 1994, 1723.; (d) M. M. Matsushita, A. Izuoka, T. Sugawara, T. Kobayashi, N. Wada, M. Ishikawa, J. Am. Chem. Soc. 1997, 119, 4369.; (e) W. Fujita, K. Awaga, Chem. Phys. Lett. 2004, 388, 186.

2 (a) K. Nakahara, S. Iwasa, M. Satoh, Y. Morioka, J. Iriyama, M. Suguro, E. Hasegawa, Chem. Phys. Lett. 2002, 359, 351.; (b) H. Nishide, S. Iwasa, Y. -J. Pu, T. Suga, K. Nakahara, M. Satoh, Electrochim. Acta. 2004, 50, 827.; (c) H. Nishide, K. Oyazu, Science. 2008, 319, 737.; (d) Y. Morita, S. Nishida, T. Murata, M. Moriguchi, A. Ueda, K. Arifuku, M. Satoh, K. Sato, T. Takui, Nat. Mater. 2011, 10, 947.

3 (a) J. M. Backer, V. G. Budker, S. I. Eremenko, Y. N. Molin, Biochim. Biophys. Acta, 1977, 460, 152.; (b) C. S. Lai, L. E. Hopwood, J. S. Hyde, S. Lukiewicz, Proc. Natl. Acad. Sci. USA, 1982, 79, 1166.; (c) D. J. Lurie, D. M. Bussell, L. H. Bell, J. R. Mallard, J. Magn. Reson. 1988, 76, 366.

4 (a) Y. Hattori, T. Kusamoto, H. Nishihara, Angew. Chem. Int. Ed. 2014, 53, 11845. (b) X. Ai, E. W. Evans, S. Dong, A. J. Gillett, H. Guo, Y. Chen, T. J. H. Hele, R. H. Friend, F. Li, Nature, 2018, 563, 536.; (c) X. Ai, Y. Chen, Y. Feng, F. Li, Angew. Chem. Int. Ed. 2018, 57, 2869.; (d) M. Chen, Y. Liao, Y. Lin, T. Xu, W. Lan, B. Wei, Y. Yuan, D. Li, X. Zhang, J. Mater. Chem. C. 2020, 8 , 14665.; (e) L. Ji, J. Shi, J. Wei, T. Yu, W. Huang, Adv. Mater. 2020, 32, 1908015.; (f) J. M. Hudson, T. J. H. Hele, E. W. Evans, J. Appl. Phys. 2021, 129, 180901.

5 (a) T. R. Carver, C. P. Slichter, Phys. Rev. 1953, 92, 212.; (b) A. Abragam, M. Goldman, Rep. Prog. Phys. 1978, 41, 395.; (c) J. H. A. -Larsen, I. Laursen, I. Leunbach, G. Ehnholm, L. -G. Wistrand, J. S. Petersson, K. Golman, J. Magn. Reson. 1998, 133, 1.; (d) Q. Z. Ni, E. Daviso, T. V. Can, E. Markhasin, S. K. Jawla, T. M. Swager, R. J. Temkin, J. Herzfeld, R. G. Griffin, Acc.
Chem. Res. 2013, 46, 1933.; (e) Y. Matsuki, T. Idehara, J. Fukazawa, T. Fujiwara, J. Magn. Reson. 2016, 264, 107.

6 Book and reviews of stable neutral radicals, see; (a) E. R. Altwicker, Chem. Rev. 1967, 67, 475.; (b) R. G. Hicks, Stable Radicals: Fundamentals and Applied Aspects of Odd-Electron Compounds, John Wiley \& Sons, Inc., 2010.; (c) K. Kato, A. Osuka, Angew. Chem. Int. Ed. 2019, 58, 8978.; (d) D. Sakamaki, S. Ghosh, S. Seki, Mater. Chem. Front. 2019, 3, 2270.

7 For typical examples of stable neutral radicals with heteroatoms, see; (a) O. A. Lebedev, S. N. Kayanovskii, Zhur. Obshch. Khim. 1960, 30, 1631.; (b) H. M. Blatter, H. Lukaszewski, Tetrahedron. Lett. 1968, 9, 2701.; (c) E. F. Ullman, J. H. Osiecki, D. G. B. Boocock, R. Darcy, J. Am. Chem. Soc. 1972, 94, 7049.; (d) Y. Morita, T. Murata, A. Ueda, C. Yamada, Y. Kanzaki, D. Shiomi, K. Sato, T. Takui, Bull. Chem. Soc. Jpn. 2018, 91, 922.

8 For several examples of stable neutral radicals with bulky substituents, see; (a) C. F. Koelsch, J. Am. Chem. Soc. 1957, 79, 4439. (b) Z. Zeng, Y. M. Sung, N. Bao, D. Tan, R. Lee, J. L. Zafra, B. S. Lee, M. Ishida, J. Ding, J. T. L. Navarrete, Y. Li, W. Zeng, D. Kim, K. -W. Huang, R. D. Webster, J. Casado, J. Wu, J. Am. Chem. Soc. 2012, 134, 14513.; (c) Y. Tian, K. Uchida, H. Kurata, Y. Hirao, T. Nishiuchi, T. Kubo, J. Am. Chem. Soc. 2014, 136, 12784.; (d) T. Nishiuchi, R. Ito, A. Takada, Y. Yasuda, T. Nagata, E. Stratmann, T. Kubo, Chem. Asian. J. 2019, 14, 1830.; (e) T. Nishiuchi, S. Aibara, T. Kubo, Angew. Chem. Int. Ed. 2018, 57, 16516.

9 For an exceptional example of a stable aromatic radical not containing a heteroatom or bulky substituent, achieved by using $\pi$-extension, see; T. Kubo Y. Katada, A. Shimizu, Y. Hirao, K. Sato, T. Takui, M. Uruichi, K. Yakushi, R. C. Haddon, J. Am. Chem. Soc. 2011, 133, 14240.

10 M. Ballester, Acc. Chem. Res. 1985, 18, 380.

11 (a) S. Andersson, F. Radner, A. Rydbeek, R. Servin, L. -G. Wistrand, US Patent, 1996, 5,530,140.; (b) T. J. Reddy, T. Iwama, H. J. Halpern, V. H. Rawal, J. Org. Chem. 2002, 67, 4635

12 P. D-. Drouhard, H. Y. V. Ching, C. Decroos, R. Guillot, Y. Li, L. C. Tabares, C. Policar, H. C. Bertrand, S. Un, Phys. Chem. Chem. Phys. 2020, 22, 20792.

13 Several TIPS-ethynyl substituted aromatic hydrocarbon radicals allow to investigate it as a stable monomer, see; (a) $Q$. Xiang, J. Guo, J. Xu, S. Ding, Z. Li, G. Li, H. Phan, Y. Gu, Y. Dang, Z. Xu, Z. Gong, W. Hu, Z. Zeng, J. Wu, Z. Sun, J. Am. Chem. Soc. 2020, 142, 11022.; (b) Q. Xiang, J. Xu, J. Guo, Y. Dang, Z. Xu, Z. Zeng, Z. Sun, Chem. Eur. J. 2021, 27, 8203.

14 S. Qiu, Y. Zhang, X. Huang, L. Bao, Y. Hong, Z. Zeng, J. Wu, Org. Lett. 2016, 18, 6018.

15 (a) R. Tanimoto, S. Suzuki, M. Kozaki, K. Okada, Chem. Lett. 2014, 43, 678.; (b) S. Suzuki, F. Nakamura, T. Naota, Mater. Chem. Front. 2018, 2, 591.; (c) K. Yamada, X. Zhang, R. Tanimoto, S. Suzuki, M. Kozaki, R. Tanaka, K. Okada, Bull. Chem. Soc. Jpn. 2018, 91, 1150.

16 S. Wu, M. Li, H. Phan, D. Wang, T. S. Herng, J. Ding, Z. Lu, J. Wu, Angew. Chem. Int. Ed. 2018, 57, 8007. 